\title{
Rehabilitation strategies for optimisation of functional recovery after major joint replacement
}

\author{
Thomas Bandholm ${ }^{1,2,3^{*}}$, Thomas W. Wainwright ${ }^{4}$ and Henrik Kehlet ${ }^{5}$
}

\begin{abstract}
Exercise-based interventions applied before and after total hip and knee arthroplasty (THA and TKA, respectively) have been investigated for a number of years, based on the assumption that they will enhance post-operative recovery. Although recent studies suggest that high-volume, pre-operative exercise may enhance post-operative recovery after TKA, studies of post-operative exercise-based interventions, have not found superiority of one exercise regime over another. It seems, however, that post-operative, exercise-based, rehabilitation is superior to no or minimal rehabilitation after THA and TKA.

The goal of this commentary is to summarize recent evidence for the efficacy of different peri-operative exercise-based interventions to enhance recovery after THA and TKA, and to propose new strategies to further enhance post-operative recovery.

There is a major need to improve functional recovery after THA and TKA. We propose a strategy of "enriched" trials where specific rehabilitation interventions are applied to different patients based on, for example, their expectations for post-operative recovery, willingness to undertake exercise and physical activity, and pre-operative functional performance.
\end{abstract}

Keywords: Hip arthroplasty, Knee arthroplasty, Rehabilitation, Physical therapy, Exercise therapy

\section{Background}

Total hip and knee arthroplasty (TKA and THA) have been quoted to be some of the most successful operations and performed in an increasing number of patients every year around the world to reduce pain and improve function (Culliford et al. 2015). However, several challenges lie ahead to improve recovery (Aasvang et al. 2015). For example, according to patient-reported outcomes (PROMs), the overall result is generally positive, although discrepancies are seen when PROMs are compared to performance-based function (Luna et al. 2017, Stevens-Lapsley et al. 2011). Also, postoperative activity levels are disappointingly low in many patients, and around $20 \%$ of patients are socially isolated

\footnotetext{
* Correspondence: thomas.quaade.bandholm@regionh.dk

${ }^{1}$ Physical Medicine \& Rehabilitation Research - Copenhagen (PMR-C), Department of Occupational and Physical Therapy, Amager-Hvidovre Hospital, University of Copenhagen, Kettegaards Alle 30, DK-2650 Hvidovre, Denmark

${ }^{2}$ Department of Orthopedic Surgery, Amager-Hvidovre Hospital, University of Copenhagen, Kettegaards Alle 30, DK-2650 Hvidovre, Denmark Full list of author information is available at the end of the article
}

following surgery (Harding et al. 2014; Smith et al. 2017). Additionally, about 5 to $20 \%$ of all patients report chronic pain after THA and TKA, respectively (Beswick et al. 2012). Given the negative physical and psychological consequences of these factors on outcomes such as all-cause mortality, return to work, and leisure activities, (Smith 2017) there is a significant rehabilitation challenge for this population.

Given these recovery problems, and the fact that there is a large early postoperative loss of knee-extension strength (30 to $80 \%$ after THA and TKA, respectively) (Kehlet 2013) major efforts have been paid towards improving post-operative recovery. Since the length of hospital stay and the overall risk of complications have been reduced with the concept of fast-track surgery or enhanced recovery programs, (Jorgensen et al. 2016) the main focus of perioperative care should now be on how to accelerate post-discharge recovery and physical rehabilitation. This work, and the rehabilitation techniques chosen, should also acknowledge that the relative causes and underlying mechanisms for early decreased muscle function are still to be 
elucidated. Further investigation into the relative role of factors such as oedema, pain, reflex inhibition, and fear, on muscle function in the immediate post-operative phase may help to guide the choice of effective rehabilitation interventions.

Therefore, this commentary will summarize the recent evidence for the efficacy of different peri-operative exercise-based interventions to enhance recovery after THA and TKA, and propose future strategies to further enhance post-operative recovery.

\section{The current state of research into rehabilitation strategies}

With respect to the current evidence for pre and post-operative exercise, we have previously argued that earlier initiated and more intense types of exercise-based physical rehabilitation modalities (e.g. progressive strength training) would likely be superior to less intense (e.g. ADL training without progressive strength training) (Bandholm and Kehlet 2012). However, such superiority has been difficult to demonstrate in both THA and TKA (Bade et al. 2017; Jakobsen et al. 2014; Mikkelsen et al. 2014). Hence, there is currently no firm evidence-base for a single type of exercise-based physical rehabilitation to enhance post-operative recovery in the "average" patient (Artz et al. 2015; Coulter et al. 2013; Haas et al. 2016; Pozzi et al. 2013; Wang et al. 2016).

However, some issues have become clearer in recent years. Firstly, while it has been difficult to demonstrate clinically relevant effects of preoperative exercise on postoperative recovery after THA and TKA (Wang et al. 2016), recent studies using supervised, pre-operative, high-volume, progressive strength training may to some extent enhance the recovery of knee-extension strength and functional performance after TKA (Calatayud et al. 2017; Skoffer et al. 2016). Secondly, exercise-based rehabilitation seems to be superior to no or minimal exercise-based rehabilitation following THA and TKA (Artz et al. 2015; Coulter et al. 2013; Pozzi et al. 2013). Thirdly, home-based physical rehabilitation, i.e. having received one or a few initial exercise instructions, seems equally effective as out-patient, supervised rehabilitation (Artz et al. 2015; Coulter et al. 2013; Pozzi et al. 2013). However, none of these interventions have solved the overall functional recovery problem after these operations. In addition, research to examine specific rehabilitation interventions for patients with known risk factors for slow or impaired functional recovery is particularly lacking.

\section{Recommendations for future research into rehabilitation strategies}

Given the economic resources spent on surgery and subsequent rehabilitation of the "average" patient, (Kjellberg and Kehlet 2016) the question is whether we should change our future cost-effective strategies for improving recovery? In this context - and living in a time with a focus on personalised medicine - we hypothesise that future interventions should be based on a more detailed preoperative characterisation of patients, including: socioeconomic status, expectations regarding recovery, preoperative pain status, psychological status, preoperative fall tendency, and leg muscle-strength status, as these may represent different groups of patients and with potential large differences in post-operative functional recovery. Work to define such groups needs to be undertaken initially in prospective interventional studies.

Consequently, the future strategy may include a specific focus on preoperative exercise (prehabilitation) in patients with reduced preoperative knee-extension strength, impaired functional performance and fall tendency. This will be challenging and so future research to accurately characterise and identify these patients preoperatively is required. Such strategies, in combination with nutritional supplementation for the identified frail/ sarcopenic patients may offer enhanced benefits, given the blunted adaptive response of these patients to exercise and nutrition, and the argument that exercise and nutrition are always better together (Phillips 2017). In addition, to exercise and nutrition, educational interventions are also a recognised component of prehabilitation (Carli and Scheede-Bergdahl 2015), and as such should also be investigated, as they may help with changing patient expectations of recovery timescales, and to facilitate patient understanding of how to progress rehabilitation whilst adhering to any surgically imposed restrictions to movements or weight bearing status.

The concept of enriched trials in such "high-risk" patients is different to previous efforts, where trials have been undertaken in "average patients" with limited effect on postoperative recovery in most trials (Bandholm and Kehlet 2012). Such studies should also incorporate methods to capture adherence rates to home exercise, as this may be a critical factor in influencing outcomes (Bollen et al. 2014). Also, preoperative exercise as part of conservative preoperative care for the "average" patient may enable better selection of surgical candidates (Skou et al. 2015).

Returning to exercise-based postoperative physical rehabilitation, it may be preferable to use home-based exercise or community-based exercise classes (which may have additional benefits in regard to social isolation and loneliness) in most patients, following one or more initial exercise instructions (Artz et al. 2015; Coulter et al. 2013; Pozzi et al. 2013). In this context, technology that can objectively monitor exercise adherence and stimulus intensity during home-based exercise is now available, (Rathleff et al. 2014) which enables documentation of exercise adherence and intensity in relation to the degree of observed recovery. Wearable technologies such as activity trackers and smartphone apps also offer much promise within this area, and 
their value in objectively assessing functional outcomes has begun to be demonstrated (Luna et al. 2017), although further objective evaluation of their specific efficacy when used as a tool to improve outcomes is still required (Bahadori et al. 2018).

Similarly, the promising results of another technology, perioperative neuromuscular electrical stimulation (NMES) requires further investigation (Spector et al. 2016) before recommendations can be provided. The potential role of NMES in accelerating the recovery of muscle function should be evaluated along with its effect using different setting parameters on symptoms such as pain and oedema. It's use immediately post-surgery (before discharge) may also offer benefits, where by it can enable a high exercise volume, with little effort, at a time point where muscle inhibition is most pronounced. In addition, in post-discharge rehabilitation, its combination with exercise interventions (non-simultaneously) has also been argued as optimal (Vanderthommen and Duchateau 2007).

For all future research of specific rehabilitation interventons, a separation should be made between a "standard" expected good recovering patient and the more difficult patients like those with a continuous subacute or persistent pain problem, those who are on preoperative opioid treatment, (Aasvang et al. 2016) pain catastrophizers, (Lunn et al. 2015) patients receiving preoperative psychopharmacological treatment (Jørgensen et al. 2015) and combined with a detailed assessment of the patient's preoperative expectations (Haanstra et al. 2012; Nakahara et al. 2015; Palazzo et al. 2014) of their postoperative functional outcome. Such studies will be crucial to understand where we should lay resources for rehabilitation, since many patients have limited daily activity after joint replacement (Harding et al. 2014) and may not be motivated for undertaking greater levels of physical activity - even for expected health gains (Smith et al. 2015). In this case, they may potentially gain little from out-patient physical rehabilitation. Consequently, future strategies should include a focus on needs as well as motivation for physical rehabilitation. There may be patients who will recover by themselves by actively engaging in simple, inexpensive, home-based rehabilitation; patients who may not want to actively engage in any type of physical rehabilitation and have no major expectations for functional recovery besides pain alleviation; and "high-risk" patients who need much more intensive and supervised physical rehabilitation.

Given the recommendations provided, an important future step may also be the transfer of recent research evidence into practical clinical guidelines, that can help to guide clinicians and support the implementation of evidence to everyday clinical practice. Such guidance does not currently exist for physical rehabilitation after THA and TKA and may be a useful adjunct to increase the impact of future clinical trials.

\section{Conclusions}

In summary, there is a continuous major need to improve functional recovery after hip and knee replacement, because studies to date have not found superiority of one exercise regime over another. However, whilst exercise-based rehabilitation seems superior to no or minimal rehabilitation after THA and TKA, future rehabilitation strategies will require us to examine different patient groups and the use of specific postoperative rehabilitation interventions based within "enriched" trial designs.

\section{Abbreviations \\ NMES: Neuromuscular electrical stimulation; PROMs: Patient reported outcome measures; THA: Total hip arthroplasty; TKA: Total knee arthroplasty \\ Authors' contributions \\ TB - Conceived the idea for the commentary, drafted the manuscript, revised it critically for content and approved the final version. TW - Drafted the manuscript, revised it critically for content and approved the final version. HK - Conceived the idea for the commentary, drafted the manuscript, revised it critically for content and approved the final version.}

Ethics approval and consent to participate

Not applicable

\section{Consent for publication}

Not applicable

\section{Competing interests}

Thomas Bandholm - has received speaker's honoraria for various enhanced recovery after surgery symposia, but has no relevant conflict of interest related to this work. The manuscript represents a narrative update on recent scientific data, all of which have been independent of industry or agencies.

The author has not been paid to write the article nor is in any way dependent on any pharmaceutical/medical device company or other agencies. Thomas Bandholm is a Physical Therapist and Exercise Physiologist, hence, a potential cognitive bias is the love of exercise.

Thomas Wainwright - has received speaker's honoraria for various enhanced recovery after surgery symposia, but has no relevant conflict of interest related to this work. The manuscript solely represents an update on recent scientific data, all of which have been independent of industry or agencies. The author has not been paid to write the article nor is in any way dependent on any pharmaceutical/medical device company or other agencies. He is a director/treasurer of The Enhanced Recovery after Surgery Society (UK) c.i.c. (not-for-profit organisation - Company No. 10932208). Henrik Kehlet - has received speaker's honoraria for various enhanced recovery after surgery symposia, but has no relevant conflict of interest related to this work. The manuscript solely represents an update on recent scientific data, all of which have been independent of industry or agencies.

\section{Publisher's Note}

Springer Nature remains neutral with regard to jurisdictional claims in published maps and institutional affiliations.

\section{Author details}

${ }^{1}$ Physical Medicine \& Rehabilitation Research - Copenhagen (PMR-C), Department of Occupational and Physical Therapy, Amager-Hvidovre Hospital, University of Copenhagen, Kettegaards Alle 30, DK-2650 Hvidovre, Denmark. ${ }^{2}$ Department of Orthopedic Surgery, Amager-Hvidovre Hospital, University of Copenhagen, Kettegaards Alle 30, DK-2650 Hvidovre, Denmark. ${ }^{3}$ Clinical Research Centre (056), Amager-Hvidovre Hospital, University of Copenhagen, Kettegaards Alle 30, DK-2650 Hvidovre, Denmark. ${ }^{4}$ Orthopaedic Research Institute, Bournemouth University, Executive Business Centre, 89 Holdenhurst Road, Bournemouth BH8 8EB, UK. ${ }^{5}$ Rigshospitalet, Copenhagen University, Section of Surgical Pathophysiology 7621, Blegdamsvej 9, DK-2100 Copenhagen, Denmark. 
Received: 10 May 2018 Accepted: 20 September 2018 Published online: 11 October 2018

\section{References}

Aasvang EK, Luna IE, Kehlet H (2015) Challenges in postdischarge function and recovery: the case of fast-track hip and knee arthroplasty. Br J Anaesth 115: 861-866. https://doi.org/10.1093/bja/aev257

Aasvang EK, Lunn TH, Hansen TB, Kristensen PW, Solgaard S, Kehlet H (2016) Chronic pre-operative opioid use and acute pain after fast-track total knee arthroplasty. Acta Anaesthesiol Scand 60:529-536. https://doi.org/10.1111/aas.12667

Artz N, Elvers KT, Lowe CM, Sackley C, Jepson P, Beswick AD (2015) Effectiveness of physiotherapy exercise following total knee replacement: systematic review and meta-analysis. BMC Musculoskelet Disord 16(15). https://doi.org/ 10.1186/s12891-015-0469-6

Bade M et al (2017) Early high-intensity versus low-intensity rehabilitation after Total knee Arthroplasty: a randomized controlled trial. Arthritis Care Res (Hoboken) 69:1360-1368. https://doi.org/10.1002/acr.23139

Bahadori S, Immins T, Wainwright TW (2018) A review of wearable motion tracking systems used in rehabilitation following hip and knee replacement 5:1-8 https://doi.org/10.1177/2055668318771816

Bandholm T, Kehlet H (2012) Physiotherapy exercise after fast-track total hip and knee arthroplasty: time for reconsideration? Arch Phys Med Rehabil 93:12921294. https://doi.org/10.1016/j.apmr.2012.02.014

Beswick AD, Wylde V, Gooberman-Hill R, Blom A, Dieppe P (2012) What proportion of patients report long-term pain after total hip or knee replacement for osteoarthritis? A systematic review of prospective studies in unselected patients. BMJ Open 2:e000435. https://doi.org/10.1136/bmjopen-2011-000435

Bollen JC, Dean SG, Siegert RJ, Howe TE, Goodwin VA (2014) A systematic review of measures of self-reported adherence to unsupervised home-based rehabilitation exercise programmes, and their psychometric properties. BMJ Open 4:e005044. https://doi.org/10.1136/bmjopen-2014-005044

Calatayud J, Casana J, Ezzatvar Y, Jakobsen MD, Sundstrup E, Andersen LL (2017) High-intensity preoperative training improves physical and functional recovery in the early post-operative periods after total knee arthroplasty: a randomized controlled trial. Knee Surg Sports Traumatol Arthrosc 25:28642872. https://doi.org/10.1007/s00167-016-3985-5

Carli F, Scheede-Bergdahl C (2015) Prehabilitation to enhance perioperative care. Anesthesiol Clin 33:17-33. https://doi.org/10.1016/j.anclin.2014.11.002

Coulter CL, Scarvell JM, Neeman TM, Smith PN (2013) Physiotherapist-directed rehabilitation exercises in the outpatient or home setting improve strength, gait speed and cadence after elective total hip replacement: a systematic review. J Physiother 59:219-226. https://doi.org/10.1016/S18369553(13)70198-X

Culliford D, Maskell J, Judge A, Cooper C, Prieto-Alhambra D, Arden NK (2015) Future projections of total hip and knee arthroplasty in the UK: results from the UK Clinical Practice Research Datalink. Osteoarthr Cartil 23(4):594-600. https://doi.org/10.1016/j.joca.2014.12.022

Haanstra TM, van den Berg T, Ostelo RW, Poolman RW, Jansma EP, Cuijpers P, de Vet HC (2012) Systematic review: do patient expectations influence treatment outcomes in total knee and total hip arthroplasty? Health Qual Life Outcomes 10:152. https://doi.org/10.1186/1477-7525-10-152

Haas R, Sarkies M, Bowles KA, O'Brien L, Haines T (2016) Early commencement of physical therapy in the acute phase following elective lower limb arthroplasty produces favorable outcomes: a systematic review and metaanalysis examining allied health service models. Osteoarthr Cartil 24:16671681. https://doi.org/10.1016/j.joca.2016.05.005

Harding P, Holland AE, Delany C, Hinman RS (2014) Do activity levels increase after total hip and knee arthroplasty? Clin Orthop Relat Res 472:1502-1511. https://doi.org/10.1007/s11999-013-3427-3

Jakobsen TL, Kehlet H, Husted H, Petersen J, Bandholm T (2014) Early progressive strength training to enhance recovery after fast-track total knee arthroplasty: a randomized controlled trial. Arthritis Care Res (Hoboken) 66:1856-1866. https://doi.org/10.1002/acr.22405

Jørgensen CC, Knop J, Nordentoft M, Kehlet H (2015) Psychiatric disorders and psychopharmacologic treatment as risk factors in elective fast-track total hip and knee arthroplasty. Anesthesiology 123:1281-1291. https://doi.org/10. 1097/ALN.0000000000000632

Jorgensen CC, Petersen MA, Kehlet H (2016) Preoperative prediction of potentially preventable morbidity after fast-track hip and knee arthroplasty: a detailed descriptive cohort study. BMJ Open 6:e009813. https://doi.org/10. 1136/bmjopen-2015-009813
Kehlet H (2013) Fast-track hip and knee arthroplasty. Lancet 11;381(9878):16001602. doi: https://doi.org/10.1016/S0140-6736(13)61003-X

Kjellberg J, Kehlet H (2016) A nationwide analysis of socioeconomic outcomes after hip and knee replacement. Dan Med J 63:A5257

Luna IE, Kehlet H, Peterson B, Wede HR, Hoevsgaard SJ, Aasvang EK (2017) Early patient-reported outcomes versus objective function after total hip and knee arthroplasty: a prospective cohort study. Bone Joint J 99-B:1167-1175. https://doi.org/10.1302/0301-620X.99B9.BJJ-2016-1343.R1

Lunn TH, Frokjaer VG, Hansen TB, Kristensen PW, Lind T, Kehlet H (2015) Analgesic effect of perioperative escitalopram in high pain catastrophizing patients after total knee arthroplasty: a randomized, double-blind, placebocontrolled trial. Anesthesiology 122:884-894. https://doi.org/10.1097/ALN. 0000000000000597

Mikkelsen LR, Mechlenburg I, Soballe K, Jorgensen LB, Mikkelsen S, Bandholm T, Petersen AK (2014) Effect of early supervised progressive resistance training compared to unsupervised home-based exercise after fast-track total hip replacement applied to patients with preoperative functional limitations. A single-blinded randomised controlled trial. Osteoarthr Cartil 22:2051-2058. https://doi.org/10.1016/.joca.2014.09.025

Nakahara H, Okazaki K, Mizu-Uchi H, Hamai S, Tashiro Y, Matsuda S, Iwamoto Y (2015) Correlations between patient satisfaction and ability to perform daily activities after total knee arthroplasty: why aren't patients satisfied? Journal of orthopaedic science: official journal of the Japanese Orthopaedic Association 20:87-92. https://doi.org/10.1007/s00776-014-0671-7

Palazzo C et al (2014) Determinants of satisfaction 1 year after total hip arthroplasty: the role of expectations fulfilment. BMC Musculoskelet Disord 15(53). https://doi.org/10.1186/1471-2474-15-53

Phillips SM (2017) Current concepts and unresolved questions in dietary protein requirements and supplements in adults. Frontiers in Nutrition 4(13). https:// doi.org/10.3389/fnut.2017.00013

Pozzi F, Snyder-Mackler L, Zeni J (2013) Physical exercise after knee arthroplasty: a systematic review of controlled trials. Eur J Phys Rehabil Med 49:877-892 https://www.ncbi.nlm.nih.gov/pmc/articles/PMC4131551/

Rathleff MS, Bandholm T, Ahrendt P, Olesen JL, Thorborg K (2014) Novel stretchsensor technology allows quantification of adherence and quality of homeexercises: a validation study. Br J Sports Med 48:724-728. https://doi.org/10. 1136/bjsports-2012-091859

Skoffer B, Maribo T, Mechlenburg I, Hansen PM, Soballe K, Dalgas U (2016) Efficacy of preoperative progressive resistance training on postoperative outcomes in patients undergoing total knee arthroplasty. Arthritis Care Res (Hoboken) 68:1239-1251. https://doi.org/10.1002/acr.22825

Skou ST, Roos EM, Laursen MB, Rathleff MS, Arendt-Nielsen L, Simonsen O, Rasmussen S (2015) A randomized, controlled trial of total knee replacement. N Engl J Med 373:1597-1606. https://doi.org/10.1056/NEJMoa1505467

Smith T (2017) "On their own": social isolation, loneliness and chronic musculoskeletal pain in older adults. Quality in Ageing and Older Adults 18: 87-92. https://doi.org/10.1108/QAOA-03-2017-0010

Smith TO, Dainty JR, MacGregor AJ (2017) Changes in social isolation and loneliness following total hip and knee arthroplasty: longitudinal analysis of the English longitudinal study of ageing (ELSA) cohort. Osteoarthr Cartil 25: 1414-1419. https://doi.org/10.1016/j.joca.2017.04.003

Smith TO, Latham S, Maskrey V, Blyth A (2015) Patients' perceptions of physical activity before and after joint replacement: a systematic review with metaethnographic analysis. Postgrad Med J 91:483-491. https://doi.org/10.1136/ postgradmedj-2015-133507

Spector P, Laufer Y, Elboim GM, Kittelson A, Stevens L, Maffiuletti NA (2016) Neuromuscular electrical stimulation therapy to restore quadriceps muscle function in patients after orthopaedic surgery: a novel structured approach. J Bone Joint Surg Am 98:2017-2024. https://doi.org/10.2106/JBJS.16.00192

Stevens-Lapsley JE, Schenkman ML, Dayton MR (2011) Comparison of selfreported knee injury and osteoarthritis outcome score to performance measures in patients after total knee arthroplasty. PM R 3541-549; quiz 549 doi:https://doi.org/10.1016/j.pmrj.2011.03.002

Vanderthommen M, Duchateau J (2007) Electrical stimulation as a modality to improve performance of the neuromuscular system. Exerc Sport Sci Rev 35(4):180-185. https://doi.org/10.1097/jes.0b013e318156e785

Wang L, Lee M, Zhang Z, Moodie J, Cheng D, Martin J (2016) Does preoperative rehabilitation for patients planning to undergo joint replacement surgery improve outcomes? A systematic review and meta-analysis of randomised controlled trials. BMJ Open 6:e009857. https://doi.org/10.1136/bmjopen-2015009857 